RAL-TR-2000-038由

CERN-TH/2001-036

February 2001

\title{
Pair production of charged Higgs scalars from electroweak gauge boson fusion
}

\author{
S. Moretti \\ Theory Division, CERN, CH-1211 Geneva 23, Switzerland
}

\begin{abstract}
We compute the contribution to charged Higgs boson pair production at the Large Hadron Collider (LHC) due to the scattering of two electroweak (EW) gauge bosons, these being in turn generated via bremsstrahlung off incoming quarks and antiquarks: $q \bar{q} \rightarrow q \bar{q} V^{*} V^{*} \rightarrow q \bar{q} H^{+} H^{-}\left(V=\gamma, Z, W^{ \pm}\right)$. We verify that the production cross section of this mode is $\tan \beta$ independent and show that it is smaller than that of $H^{+} H^{-}$production via $q \bar{q}$-initiated processes but generally larger than that of the loop-induced channel $g g \rightarrow H^{+} H^{-}$.
\end{abstract}

\footnotetext{
${ }^{1}$ Formerly at: Rutherford Appleton Laboratory, Chilton, Didcot, Oxon OX11 0QX, UK.
} 
A charged scalar state does not belong to the particle spectrum of the Standard Model (SM). Therefore, to detect a signal of it would definitely confirm the existence of New Physics. A framework that can naturally accommodate such a particle is the Minimal Supersymmetric Standard Model (MSSM). This is a realisation of a general two Higgs Doublet Model (2HDM) within the theoretical framework provided by Supersymmetry (SUSY). While the SM incorporates only one 'neutral' Higgs boson, $\phi$, the MSSM predicts a pair of 'charged' Higgs bosons $H^{ \pm}$along with three 'neutral' ones, the CP-even $H$ and $h$ and the CP-odd $A$ [1].

It is then not surprising the considerable interest, that has revived lately [2, 3], in accessing the Higgs sector of the MSSM at the future CERN collider through the detection of charged Higgs boson states. In fact, one may well conjecture that, even in presence of a clear signal of a neutral Higgs particle, it could be difficult to distinguish between the SM and the lightest MSSM Higgs boson. For example, in the so-called 'decoupling regime' of the MSSM, one has that the $h$ couplings to ordinary matter become similar to those of the SM $\phi$ and, besides, the MSSM Higgs masses are such that $M_{h} \ll M_{H} \approx M_{A} \approx M_{H^{ \pm}}$. In fact, such a decoupling scenario occurs for $M_{H^{ \pm}} \gtrsim 150-200 \mathrm{GeV}$, for any value of $\tan \beta$. Under these circumstances, it would probably be equally challenging to detect a second neutral Higgs signal, as it would be to select a charged Higgs boson signature. This scenario, though not to be expected necessarily, may be viewed as not at all unreasonable, especially taking into account the latest LEP2 results on the possible existence of a neutral Higgs state with mass of about 110-115 GeV [4] ( $M_{h}$ in the MSSM), which, using the MSSM Higgs mass relations (now, known at two-loops [5]), implies an indirect lower bound on $M_{H^{ \pm}}$already at $140 \mathrm{GeV}$ in the low $\tan \beta$ region, say, around 3 or so․

At the LHC, light charged Higgs scalars (i.e., with $M_{H^{ \pm}}<m_{t}$ ) can be produced either in top decays, $t \rightarrow b H^{+}$(with the top quarks being mainly produced via $g g \rightarrow t \bar{t}$ ), or in pair from quark-antiquark annihilations, $q \bar{q} \rightarrow H^{+} H^{-}$[6] Heavy charged Higgs bosons (i.e., with $M_{H^{ \pm}}>m_{t}$ - those beyond the reach of the Tevatron) are mainly generated via the reaction $g \bar{b} \rightarrow \bar{t} H^{+}$[9]. (In fact, the two processes, $g g \rightarrow t \bar{t}$, with $t \rightarrow b H^{+}$, and $g \bar{b} \rightarrow \bar{t} H^{+}$, can be connected [10] by looking at the generic subprocess $g g \rightarrow \bar{t} b H^{+}$[11].) Alternative production channels at the LHC are, for heavy $M_{H^{ \pm}}$: the $b q \rightarrow b H^{ \pm} q^{\prime}$ mode

\footnotetext{
${ }^{2}$ Here, $\tan \beta$ denotes the ratio of the vacuum expectation values of the two Higgs doublets of the MSSM and can conveniently be used to parametrise at tree level the entire Higgs sector, alongside one of the masses, e.g., $M_{H^{ \pm}}$itself.

${ }^{3}$ The direct experimental limit obtained at LEP2 on $M_{H^{ \pm}}$, based on searches for $e^{+} e^{-} \rightarrow H^{+} H^{-}$events, is at present much smaller: just below $M_{W \pm}$ [4].

${ }^{4}$ At the forthcoming Run II of the upgraded Fermilab Tevatron, the first of these channels will allow experimenters to scan the MSSM parameter space for large and small values of $\tan \beta$, say, below 1.5 and above $m_{t} / m_{b}$, roughly up to the kinematical limit of the $t \rightarrow b H^{+}$decay, $m_{t}-m_{b}$ [7], whereas the second channel will be useful in the intermediate $\tan \beta$ region 8, provided charged Higgs bosons are light enough, as simple phase space suppression severely handicaps pair production at $\sqrt{s}=2 \mathrm{TeV}$.
} 
of Ref. [12]; again, charged Higgs boson pair production, but now supplemented by the loop-induced subprocesses $g g \rightarrow H^{+} H^{-}$[13]]-[15]; associated production $g g, b \bar{b} \rightarrow W^{ \pm} H^{\mp}$ [16] and $q \bar{q}^{\prime} \rightarrow \Phi H^{ \pm}$, with $\Phi=h, H$ and $A$ [6].

If one makes the assumption that the typical mass scale of sparticle states is much higher than $M_{H^{ \pm}}$(e.g., $M_{\text {SUSY }}=1 \mathrm{TeV}$ - as we do throughout the paper), thus preventing the decay of charged Higgs bosons via SUSY channels, the decay signature of $H^{ \pm}$bosons is fairly model independent and dominated by four decay channels at most [17]: $H^{+} \rightarrow t \bar{b}$, $H^{+} \rightarrow \tau^{+} \nu_{\tau}, H^{+} \rightarrow c \bar{s}$ and $H^{+} \rightarrow W^{+} h$. By exploiting the above production channels in conjunction with these decay modes, it has been shown that $H^{ \pm}$scalars with masses up to $400 \mathrm{GeV}$ can be discovered at the LHC if $\tan \beta \lesssim 3$ (which is in the neighborhood of the indirect limit from LEP2) or $\tan \beta \gtrsim 10-15$ (with the minimum occurring when $M_{H^{ \pm}}$is close to $m_{t}$ ). Alternatively, if one allows for the contribution of SUSY decay modes, according to Ref. [18], the surviving region $3 \lesssim \tan \beta \lesssim 10$ (with $M_{H^{ \pm}} \lesssim 400 \mathrm{GeV}$ ) should adequately be covered by resorting to the decays $H^{ \pm} \rightarrow \tilde{\chi}_{1}^{ \pm} \tilde{\chi}_{1}^{0}$ and $H^{ \pm} \rightarrow \tilde{\chi}_{1}^{ \pm} \tilde{\chi}_{2}^{0}, \tilde{\chi}_{1}^{ \pm} \tilde{\chi}_{3}^{0}$, i.e., into combinations of chargino-neutralino pairs?.

Once charged Higgs bosons will have been detected through the leading production channels, and their mass measured, the emphasis will turn to studying their properties. In fact, with the high luminosity of the LHC, also the various subleading production processes would be established experimentally. Among these, it is the $g g \rightarrow H^{+} H^{-}$channel, originally discussed in Ref. [13], that has gathered considerable attention in the recent years [14, 15]. The reason is twofold. Firstly, it in principle allows one to measure directly the strength of the trilinear vertices between charged and neutral CP-even Higgs bosons, $h H^{+} H^{-}$and $\mathrm{HH}^{+} \mathrm{H}^{-}$, thus shading light on the structure of the Higgs sector of the MSSMF. The determination of these couplings is in fact a necessary step in reconstructing the self-interaction terms in the full Higgs potential. In contrast, in all other processes mentioned, only gauge $\left(\gamma H^{+} H^{-}, Z H^{+} H^{-}, h W^{+} H^{-}, H W^{+} H^{-}\right.$and $\left.A W^{+} H^{-}\right)$and Yukawa $\left(t \bar{b} H^{-}\right)$couplings can be accessed. Secondly, effects of SUSY could be manifest in the $g g \rightarrow H^{+} H^{-}$channel, because of virtual squark loops entering the production stage, even when charged Higgs bosons are too light to decay directly into sparticles. Unfortunately, from these points of views, the $g g \rightarrow H^{+} H^{-}$subprocess is biased by the presence of the $q \bar{q} \rightarrow H^{+} H^{-}$channel, whose production rates are much larger at the LHC (a sizable contribution comes from the previously overlooked $b \bar{b} \rightarrow H^{+} H^{-}$channel, as pointed out recently in the first paper of Ref. [14], particularly at large $\tan \beta$ ), and a complete phenomenological analysis on how to disentangle the two is still lacking.

The purpose of this paper is to show that, at the LHC, pairs of charged Higgs bosons can also be produced via the fusion of gauge vector bosons, $\gamma, Z$ and $W^{ \pm}$, at rates comparable

\footnotetext{
${ }^{5}$ According to the typical $H^{ \pm}$decay rates found in [18], one should not expect the scope of the SM channels to be spoiled by the presence of the new SUSY modes.

${ }^{6} \mathrm{As}$ a result of $\mathrm{CP}$-invariance, there exists no $\mathrm{AH}^{+} \mathrm{H}^{-}$coupling at tree level.
} 
to those induced by parton fusion in $q \bar{q}$ and $g g$ scatterings. Here, the gauge bosons are emitted by incoming quarks and antiquarks, and the all process can be sketched as follows:

$$
q \bar{q} \rightarrow q \bar{q} V^{*} V^{*} \rightarrow q \bar{q} H^{+} H^{-} \quad\left(V=\gamma, Z, W^{ \pm}\right)
$$

where $q$ refers to both quarks and antiquarks (of any possible flavour) in the appropriate combinations?. The Feynman diagrams involved can be found in Fig. 1. However, notice that not all of these appear for each quark flavour combination. (For example, for $u \bar{u} \rightarrow$ $u \bar{u} H^{+} H^{-}$no $W^{ \pm}$mediated diagrams enter whereas for $u \bar{u} \rightarrow d \bar{d} H^{+} H^{-}$the latter are needed.) Besides, we have not shown the graphs that differ from those depicted in Fig. 1 only in the exchange of a fermion leg, as it happens when identical flavours appear in the initial and final states. The matrix element (ME) of process (1) has been computed by means of helicity amplitude techniques. Furthermore, it has been checked for gauge and BRS invariance and integrated numerically over a four-body phase space.

The total cross section for process (1) at the $\mathrm{LHC}(\sqrt{s}=14 \mathrm{TeV})$, before any acceptance cuts, can be found in Fig. 2 (continuous line), compared to the yield of the other two production processes of charged Higgs boson pairs, i.e., $q \bar{q} \rightarrow H^{+} H^{-}$and $g g \rightarrow H^{+} H^{-}$, for three reference values of $\tan \beta$ and with $M_{H^{ \pm}}$in the range 130 to $400 \mathrm{GeV}$. Notice that in order to obtain a finite answer for process (1) (in the case of photon exchange), we have adopted a non-zero value for the mass of all quark flavours. We have chosen $m_{u}=m_{d}=0.32$ $\mathrm{GeV}, m_{s}=0.50 \mathrm{GeV}, m_{c}=1.55 \mathrm{GeV}$ and $m_{b}=4.25 \mathrm{GeV}$. As for the mass of the neutral Higgs bosons, we have used the Renormalisation Group ( $\mathrm{RG}$ ) improved one-loop relations of Ref. [20], for the case of vanishing 'mixing parameters' (i.e., $\mu=A_{b}=A_{t}=0$ ). Similarly, we have proceeded for the Higgs couplings.

Contrary to the $q \bar{q} \rightarrow H^{+} H^{-}$and $g g \rightarrow H^{+} H^{-}$channels, process (1) shows no visible $\tan \beta$ dependence, because of the tiny contribution of the $H, h$ and $A$ mediated graphs (numbers 15,16 and 18 in Fig. 11). In the first process, such a dependence is induced by $b \bar{b} \rightarrow H^{+} H^{-}$scatterings, whereas in the second one, it enters via both the triangle and the box quark-graphs: see Fig. 1 of Ref. [15]. The overall rate of process (1) is generally larger than that of $g g \rightarrow H^{+} H^{-}$(except for very large $\tan \beta$ values) but smaller than that of $q \bar{q} \rightarrow H^{+} H^{-}$, though asymptotically (i.e., for very large values of $\left.M_{H^{ \pm}}\right), q \bar{q} \rightarrow q \bar{q} H^{+} H^{-}$ approaches $q \bar{q} \rightarrow H^{+} H^{-}$. For an annual integrated luminosity of 100 inverse femtobarns, something like 1500 to 150 events of the type (1) could be produced per experiment at the LHC, for charged Higgs boson masses ranging from 140 to $400 \mathrm{GeV}$.

\footnotetext{
${ }^{7}$ For simplicity, we have taken the Cabibbo-Kobayashi-Maskawa mixing matrix to be diagonal.

${ }^{8}$ For reference, hereafter, we use the MRS(LO05A) [19] Parton Distribution Functions (PDFs), with factorisation/renormalisation scale $Q=\mu=\sqrt{\hat{s}}$, i.e., the centre-of-mass (CM) energy at parton level.

${ }^{9}$ Here, for simplicity, we are not including the effects of squark loops in $g g \rightarrow H^{+} H^{-}$: again, see Fig. 1 of 15. These can significantly enhance the corresponding cross section, e.g., by up to $50 \%$, depending upon $\tan \beta$, in a Minimal Supergravity (MSUGRA) inspired scenario.
} 
Fig. 3 presents the LHC cross section of the single $H^{ \pm}$-production mechanisms discussed above, for $M_{H^{ \pm}} \gtrsim m_{t}$ and our usual three choices of $\tan \beta$. (Here, we have plotted $g g \rightarrow \bar{t} b H^{+}$, accounting for the aforementioned top-antitop production and decay as well as the $b g$-fusion channel, with the normalisation advocated in Ref. [10].) Clearly, by comparing Fig. 2 to Fig. 3 (notice the different normalisation), one realises that process (1) is never dominant, although, at $\tan \beta=7$, it is just above one order of magnitude smaller than the dominant $g g \rightarrow \bar{t} b H^{+}$mode. The reason of the drop in production rates of the latter process, similarly to what happens for $b q \rightarrow b H^{ \pm} q^{\prime}$ and $b \bar{b}, g g \rightarrow W^{ \pm} H^{\mp}$, see Fig. 通, is due to a coupling of the form

$$
\sim \frac{g}{2 \sqrt{2} M_{W^{ \pm}}} H^{+}\left(m_{t} \cot \beta \bar{t} b_{L}+m_{b} \tan \beta \bar{t} b_{R}\right)
$$

whose square - entering the corresponding production cross sections - has a minimum at $\tan \beta \simeq 7$ (this is indeed the reason of the similar trend seen in the previous figure for $b \bar{b} \rightarrow H^{+} H^{-}$and $g g \rightarrow H^{+} H^{-}$as well).

The independence of $\tan \beta$ is an attractive feature that could in principle render process (1) an interesting discovery channel of charged Higgs bosons, complementary to all other modes proportional to the square of the expression in eq. (2) $⿴$. In fact, a simple measurement of the total cross section $\sigma\left(q \bar{q} \rightarrow q \bar{q} H^{+} H^{-} \rightarrow q \bar{q} X\right)$, above the SM rates, would suffice to estimate an $M_{H^{ \pm}}$value, that could then be employed in background suppression in some suitable Higgs decay channel. In order to attempt a Higgs mass reconstruction, we proceed as follows. First, we exploit the presence of two forward jets in the final state of process (11), that can be used for tagging purposes and QCD background suppression, pretty much in the same spirit as in Ref. [21] (see also [22]). There, it was shown how, in the SM Higgs process

$$
q \bar{q} \rightarrow q \bar{q} V^{*} V^{*} \rightarrow q \bar{q} \phi \rightarrow q \bar{q} W^{+} W^{-},
$$

proceeding via $W^{+} W^{-}$and $Z Z$ fusion $\left(V=Z, W^{ \pm}\right)$, the selection of the two (rather forward) quark-jets in the final state, within a detector acceptance region defined by $p_{T}^{j}>20$ $\mathrm{GeV}$ and $\left|\eta^{j}\right|<5$, can aid to strongly reduce the overwhelming (but rather central) QCD background in $t \bar{t} j j$ and $W^{+} W^{-} j j$ events, where $j$ represents a jet, thus rendering process (3) a viable mechanism to detect SM Higgs signals via leptonic $W^{+} W^{-}$decays section of process (11), after the above transverse-momentum and pseudorapidity cuts are

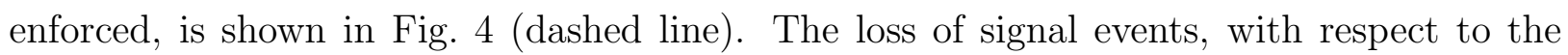
total rate (continuous line), is rather contained (around $35 \%$, typically), owning to the fact that for $M_{H^{ \pm}} \gtrsim 130 \mathrm{GeV}$ the bulk of the production rates is due to $W^{+} W^{-}$and $Z Z$ fusion,

\footnotetext{
${ }^{10}$ The $\tan \beta$ dependence of the $q \bar{q}^{\prime} \rightarrow \Phi H^{ \pm}$processes, with $\Phi=h, H$ and $A$, is less straightforward than in eq. (2), as all these channels also proceed via $\Phi q \bar{q}, \Phi W^{ \pm} H^{\mp}$ and $\Phi H^{ \pm} H^{\mp}$ vertices, which are more complicated functions of $\tan \beta$, involving in particular the Higgs 'mixing angle' $\alpha$.

${ }^{11}$ In fact, the decay $W^{+} W^{-} \rightarrow \ell^{+} \ell^{-} \nu_{\ell} \bar{\nu}_{\ell}$ represents the best way to extract the SM Higgs signal from the mentioned backgrounds [23, 24] over the mass region between 130 and $180 \mathrm{GeV}$ or so.
} 
rather than to $\gamma \gamma$ and $\gamma Z$, which would dominate at smaller values of $M_{H^{ \pm}}$.

A tentative selection procedure of the mass resonance in the signal could be the one sketched below. Notice that we carry out our analysis at parton level only, thus neglecting parton shower and hadronisation effects, although we account for typical detector resolutions, as the transverse momenta of all visible particles in the final state have been smeared according to a Gaussian distribution, with $\left(\sigma\left(p_{T}\right) / p_{T}\right)^{2}=\left(0.6 / \sqrt{p_{T}}\right)^{2}+(0.04)^{2}$ for all jets and $\left(\sigma\left(p_{T}\right) / p_{T}\right)^{2}=\left(0.12 / \sqrt{p_{T}}\right)^{2}+(0.01)^{2}$ for the leptons. The missing transverse momentum has been evaluated from the vector sum of the jet and lepton transverse momenta after resolution smearing.

1. We ask for the decays $H^{+} \rightarrow t \bar{b} \rightarrow b \bar{b} W^{+}$and $H^{-} \rightarrow \tau^{-} \bar{\nu}_{\tau}$, and charge conjugate cases, with the $W^{+}$decaying hadronically to a pair of light jets. At the same time, one requires to tag the $\tau^{-}$via its leptonic or hadronic decay channels ${ }^{[2}$. Hence, the final signature is ' 6 jets $+\tau^{ \pm}+$missing energy', with two of the jets being initiated by $b$-quarks. The largest background to this signature is most probably due to $q \bar{q}, g g \rightarrow t \bar{t} g g$ events, with $t \rightarrow b W^{+}$and $\bar{t} \rightarrow \bar{b} \tau^{-} \bar{\nu}_{\tau}$, with the two gluons yielding low transverse momentum jets in the forward and backward directions.

2. We impose the mentioned transverse momentum and pseudorapidity constraints on all six jets: $p_{T}^{j}>20 \mathrm{GeV}$ and $\left|\eta^{j}\right|<5$. Besides, we require that the difference in pseudorapidity between the two quark/gluon-jets with highest and lowest $\eta$-value is larger than 2: $\left|\eta_{\max }^{j}-\eta_{\min }^{j}\right| \equiv\left|\eta^{j_{1}}-\eta^{j_{2}}\right|>2$.

3. Leptons (electrons and/or muons) are accepted if $p_{T}^{\ell}>20 \mathrm{GeV}$ and $\left|\eta^{\ell}\right|<2.5$.

4. A pseudorapidity-azimuthal separation between any jet-jet and jet-lepton pair is imposed: $\Delta R \equiv \sqrt{\Delta \eta^{2}+\Delta \Phi^{2}} \geq 0.7$.

5. A threshold on the missing transverse energy is enforced too: $p_{T}^{\text {miss }}>M_{H^{ \pm}} / 2$.

6. We ask that two quark/gluon-jets (among those not satisfying the last requirement in 1.) reproduce the $W^{ \pm}$mass within $10 \mathrm{GeV}:\left|M_{j_{3} j_{4}}-M_{W^{ \pm}}\right|<10 \mathrm{GeV}$.

7. We further ask that the two above jets reproduce the top mass within $25 \mathrm{GeV}$, if paired with a third quark/gluon-jet: $\left|M_{j_{3} j_{4} j_{5}}-m_{t}\right|<25 \mathrm{GeV}$.

8. We impose the veto $\left|M_{j_{6} \tau \nu_{\tau}}-m_{t}\right|>25 \mathrm{GeV}$, where $M_{j_{6} \tau \nu_{\tau}}$ is the invariant mass obtained by combining the remaining quark/gluon-jet with the $\tau$-momentum and the one of the parent neutrino, the latter being reconstructed by adopting the technique outlined in the fourth paper of [2].

\footnotetext{
${ }^{12}$ We assume that the latter can efficiently be distinguished from the shower of an (anti)quark or gluon.
} 
9. We also cut di-jet invariant masses obtained from the two jets already identified in 1 . which are below the charged Higgs mass: i.e., $M_{j_{1} j_{2}}>M_{H^{ \pm}}$.

10. Finally, we plot the invariant mass of the four-jet system recoiling against the $j_{1} j_{2}$ and $\tau^{ \pm}$-neutrino pairs.

Although the number of events of type 1. produced via process (1) can still be sizable at the end of the sequence of cuts in 2.-8., and the charged Higgs mass can be reconstructed rather neatly via step 9., see Fig. 5, the background from the QCD events $q \bar{q}, g g \rightarrow t \bar{t} g g$ is prohibitive. Despite having been reduced by several orders of magnitude, it overwhelms the Higgs resonances completely. It should in fact be noticed that the integral over the three Higgs curves in Fig. 5, multiplied by the mentioned annual luminosity (i.e., $100 \mathrm{fb}^{-1}$ ), yields only 6, 4 and 2 events, in correspondence of $M_{H^{ \pm}}=215,310$ and $408 \mathrm{GeV}$, respectively, whereas the background rates sum up to a total which is typically 1000 times bigger in the vicinity of the peaks. Besides, the above numbers for the $q \bar{q} \rightarrow q \bar{q} H^{+} H^{-}$process are obtained for $\tan \beta=40$, value for which the product of the branching ratios of the two channels $H^{+} \rightarrow t \bar{b} \rightarrow b \bar{b} W^{+}$and $H^{-} \rightarrow \tau^{-} \bar{\nu}_{\tau}$ is maximal, within the theoretically preferred $\tan \beta$ interval, i.e., $\tan \beta \lesssim m_{t} / m_{b}$. The situation does not improve substantially for other values of $M_{H^{ \pm}}$in the heavy mass range or other, more selective choices of cuts, than those illustrated here.

In summary, we have demonstrated that $\mathrm{H}^{+} \mathrm{H}^{-}$production can be induced at the LHC by three distinct processes: quark-antiquark, (electroweak) vector-vector and gluon-gluon fusion, in order of quantitative importance. Whereas the phenomenological relevance of the first and third of these modes has been recognised for some time (and stressed again recently), that of the second channel constitutes the novelty of our research. Besides, at the LHC, the $g g$-mode has been advocated as one of the best ways to probe the $h H^{+} H^{-}$and $\mathrm{HH}^{+} \mathrm{H}^{-}$couplings among Higgs scalars and as an effective means to constrain the squark sector of SUSY, as both neutral Higgs bosons and scalar quarks enter the virtual stages of the production process. Hence, in suppressing the background to $g g \rightarrow H^{+} H^{-}$, special care has to be adopted in dealing not only with the $q \bar{q} \rightarrow H^{+} H^{-}$mode, but also with the $q \bar{q} \rightarrow q \bar{q} H^{+} H^{-}$channel. Finally, the EW vector-vector fusion reaction has been tested as a possible discovery mode of heavy charged Higgs bosons, as a complement to the leading $g g \rightarrow \bar{t} b H^{+}$channel in the $3 \lesssim \tan \beta \lesssim 10$ window, where the discovery potential of the latter is seriously hampered by a steeply falling production rate (with a minimum at $\tan \beta=7$ ). Despite the independence of $q \bar{q} \rightarrow q \bar{q} H^{+} H^{-}$from $\tan \beta$ could allow for a prompt estimate of $M_{H^{ \pm}}$and the consequent mass resonance selection can be made viable, the background from QCD induced events of the type $q \bar{q}, g g \rightarrow t \bar{t} g g$ is prohibitively large, at least in the channel $H^{+} H^{-} \rightarrow$ '4 jets $+\tau^{ \pm}+$missing energy', including the forward tagging of the two quark-jets produced in association with the Higgs boson pair. Indeed, if the signature that we have chosen is to be useful in searching for pairs of charged Higgs bosons at the 
LHC, far better cuts than those designed here will need to be devised. An alternative, cleaner detection mode could be $H^{+} H^{-} \rightarrow \tau^{+} \nu_{\tau} \tau^{-} \bar{\nu}_{\tau}$, however, this requires more realistic simulations (including double $\tau^{ \pm}$reconstruction in a real detector environment) than those that can be carried out in a parton level study.

\section{Acknowledgements}

We are grateful to PPARC for financial support and to the Theory Group at the Rutherford Appleton Laboratory (RAL) for hospitality during the early stages of this work. We also thank Kosuke Odagiri for suggesting the topic of this research, for innumerable helpful discussions and for numerical comparisons as well.

\section{References}

[1] J.F. Gunion, H.E. Haber, G.L. Kane and S. Dawson, "The Higgs Hunters' Guide" (Addison-Wesley, Reading, MA, 1990).

[2] S. Raychaudhuri and D.P. Roy, Phys. Rev. D52 (1995) 1556, Phys. Rev. D53 (1996) 4902; J.F. Gunion, Phys. Lett. B322 (1994) 125; V. Barger, R.J.N. Phillips and D.P. Roy, Phys. Lett. B324 (1994) 236; K. Odagiri, preprint RAL-TR-1999-012, January 1999, hep-ph/9901432, Phys. Lett. B452 (1999) 327; D.P. Roy, Phys. Lett. B459 (1999) 607; D.J. Miller, S. Moretti, D.P. Roy and W.J. Stirling, Phys. Rev. D61 (2000) 055011; S. Moretti and D.P. Roy, Phys. Lett. B470 (1999) 209; M. Drees, M. Guchait and D.P. Roy, Phys. Lett. B471 (1999) 39; S. Moretti, Phys. Lett. B481 (2000) 49.

[3] CMS Technical Proposal, CERN/LHCC/94-38 (1994); ATLAS Technical Proposal, CERN/LHCC/94-43 (1994); K.A. Assamagan, preprint ATL-PHYS-99-013, preprint ATL-PHYS-99-025; K.A. Assamagan and Y. Coadou, preprint ATL-COM-PHYS-2000017; K.A. Assamagan, A. Djouadi, M. Drees, M. Guchait, R. Kinnunen, J.L. Kneur, D.J. Miller, S. Moretti, K. Odagiri and D.P. Roy, contribution to the Workshop 'Physics at TeV Colliders', Les Houches, France, 8-18 June 1999, preprint PM/00-03, pages 3653, February 2000, hep-ph/0002258 (to appear in the proceedings).

[4] LEP Higgs Working Group, http://www.cern.ch/LEPHIGGS/.

[5] S. Heinemeyer, W. Hollik and G. Weiglein, Eur. Phys. J. C9 (1999) 343.

[6] E. Eichten, I. Hinchliffe, K. Lane and C. Quigg, Rev. Mod. Phys. 56 (1984) 579. 
[7] See, e.g.: M. Carena, J. Conway, H.E. Haber and J. Hobbs, 'Report of the Higgs Working Group', to appear in the Proceedings of the 'Tevatron Run II SUSY/Higgs Workshop', February-November 1998, Fermilab, Batavia, Illinois (unpublished).

[8] K. Odagiri, private communication.

[9] J.F. Gunion, H.E. Haber, F.E. Paige, W.-K. Tung and S.S.D. Willenbrock, Nucl. Phys. B294 (1987) 621.

[10] F. Borzumati, J.-L. Kneur and N. Polonsky, Phys. Rev. D60 (1999) 115011.

[11] J.L. Diaz-Cruz and O.A. Sampayo, Phys. Rev. D50 (1994) 6820.

[12] S. Moretti and K. Odagiri, Phys. Rev. D55 (1997) 5627.

[13] S.S.D. Willenbrock, Phys. Rev. D35 (1987) 173.

[14] A.A. Barrientos Bendezú and B.A. Kniehl, Nucl. Phys. B568 (2000) 305; A. Krause, T. Plehn, M. Spira and P.M. Zerwas, Nucl. Phys. B519 (1998) 85; Y. Jiang, W.-G. Ma, L. Han, M. Han and Z.-H. Yu, J. Phys. G23 (1997) 385, Erratum, ibidem G23 (1997) 1151; O. Brein and W. Hollik, Eur. Phys. J. C13 (2000) 175.

[15] Y. Jiang, W.-G. Ma, L. Han, M. Han and Z.-H. Yu, J. Phys. G24 (1998) 83.

[16] D.A. Dicus, J.L. Hewett, C. Kao and T.G. Rizzo, Phys. Rev. D40 (1989) 787; A.A. Barrientos Bendezú and B.A. Kniehl, Phys. Rev. D59 (1999) 015009, ibidem D61 (2000) 097701, preprint DESY 00-110, MPI-PhT/2000-27, July 2000, hep-ph/0007336; S. Moretti and K. Odagiri, Phys. Rev. D59 (1999) 055008.

[17] S. Moretti and W.J. Stirling, Phys. Lett. B347 (1995) 291, Erratum, ibidem B366 (1996) 451; A. Djouadi, J. Kalinowski and P.M. Zerwas, Z. Phys. C70 (1996) 435; E. Ma, D.P. Roy and J. Wudka, Phys. Rev. Lett. 80 (1998) 1162.

[18] M. Bisset, M. Guchait and S. Moretti, preprint DESY 00-150, TUHEP-TH-00124, RAL-TR-2000-029, October 2000, hep-ph/0010253.

[19] A.D. Martin, R.G. Roberts, W.J. Stirling and R.S. Thorne, Phys. Lett. B443 (1998) 301.

[20] H.E. Haber, R. Hempfling and A.H. Hoang, Z. Phys. C75 (1997) 539; M. Carena, J. Espinosa, M. Quiros and C.E.M. Wagner, Phys. Lett. B355 (1995) 209.

[21] D. Rainwater and D. Zeppenfeld, Phys. Rev. D60 (1999) 113004, Erratum, ibidem D61 (2000) 099901. 
[22] T. Plehn, D. Rainwater and D. Zeppenfeld, Phys. Rev. D61 (2000) 093005.

[23] M. Dittmar and H. Dreiner, Phys. Rev. D55 (1997) 167.

[24] S. Moretti, Phys. Rev. D56 (1997) 7427. 


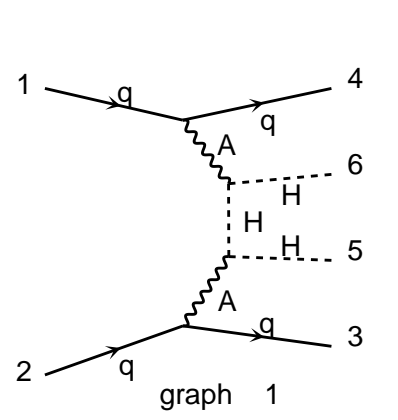

Diagrams by FEYNMAN DRAW
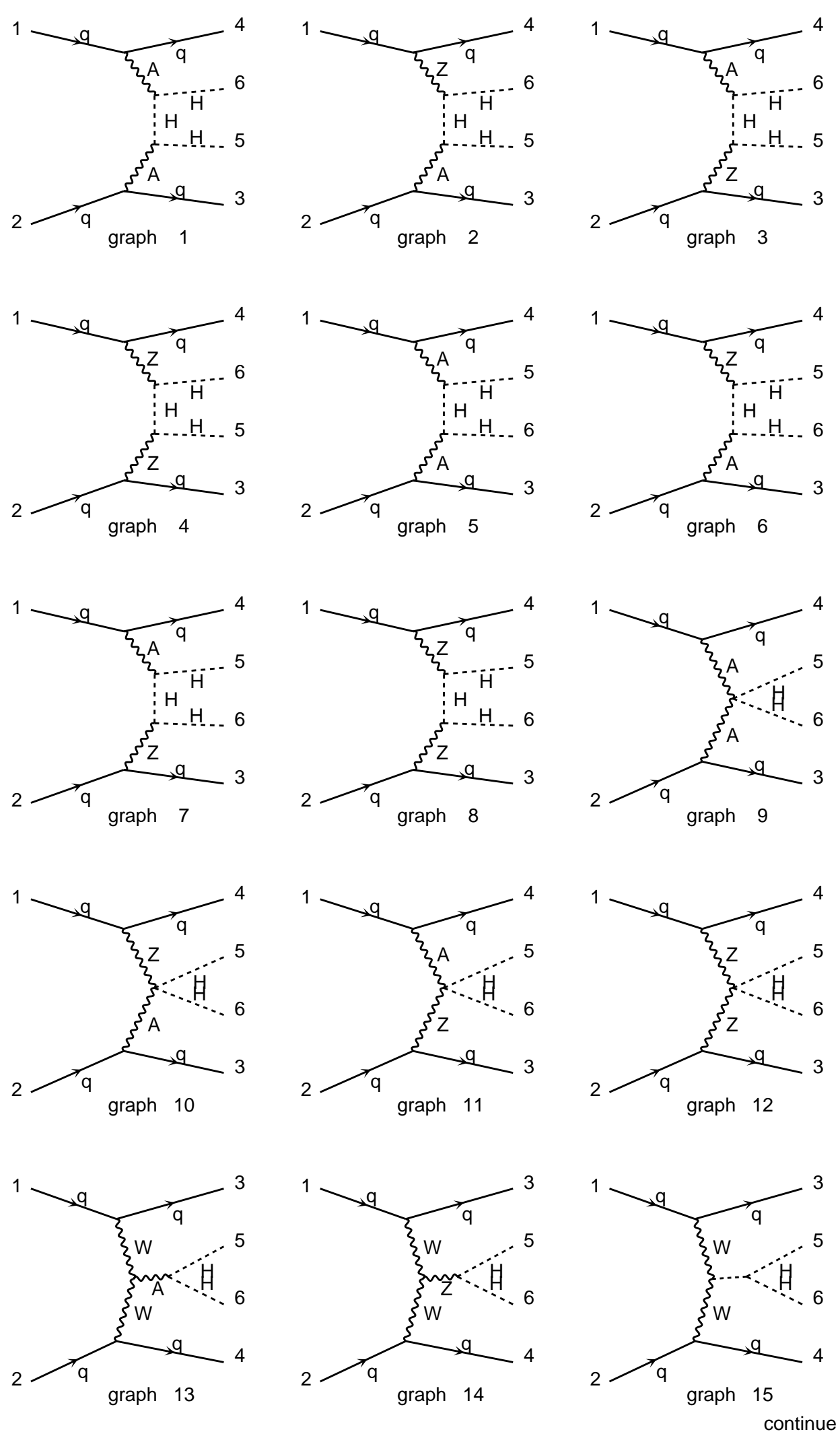


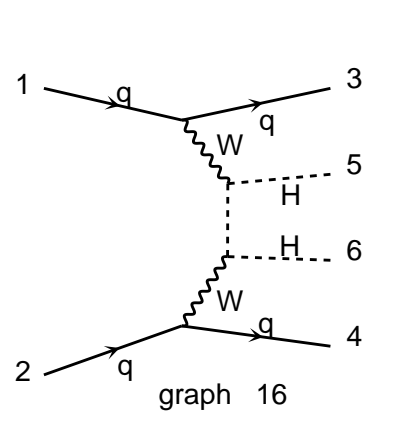

\section{Diagrams by FEYNMAN DRAW}
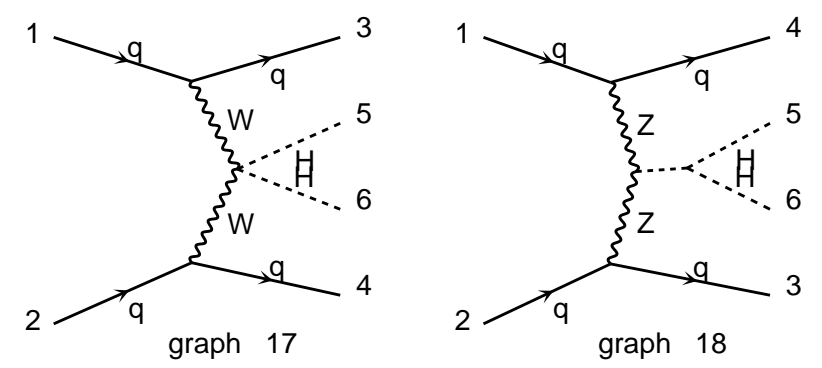

Figure 1: Feynman diagrams at tree level for process (11). The labels $\mathrm{q}, \mathrm{A}, \mathrm{Z}, \mathrm{W}$ and $\mathrm{H}$ refer to a (anti)quark, $\gamma, Z, W^{ \pm}$and $H^{ \pm}$boson, respectively, whereas an unlabelled, internal dashed line represents a summation over $H, h$ and $A$ boson propagators. 


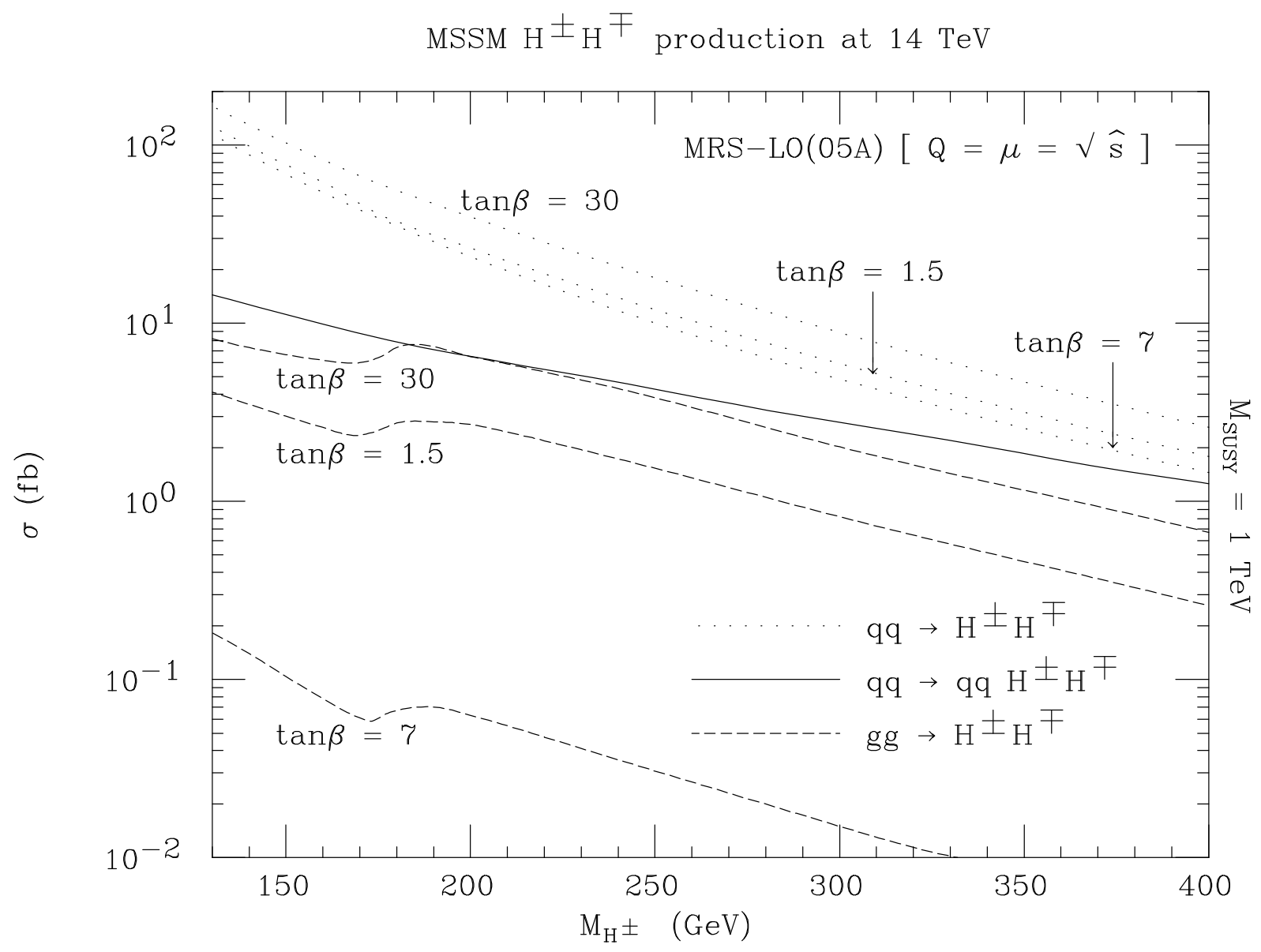

Figure 2: Cross section in femtobarns at the LHC for the three $H^{+} H^{-}$production processes discussed in the text, $q \bar{q} \rightarrow H^{+} H^{-}$(including the $b \bar{b}$ contribution), $g g \rightarrow H^{+} H^{-}$and $q \bar{q} \rightarrow q \bar{q} H^{+} H^{-}$, for $\tan \beta=1.5,7$ and 30. In the last process, there is no visible $\tan \beta$ dependence. 

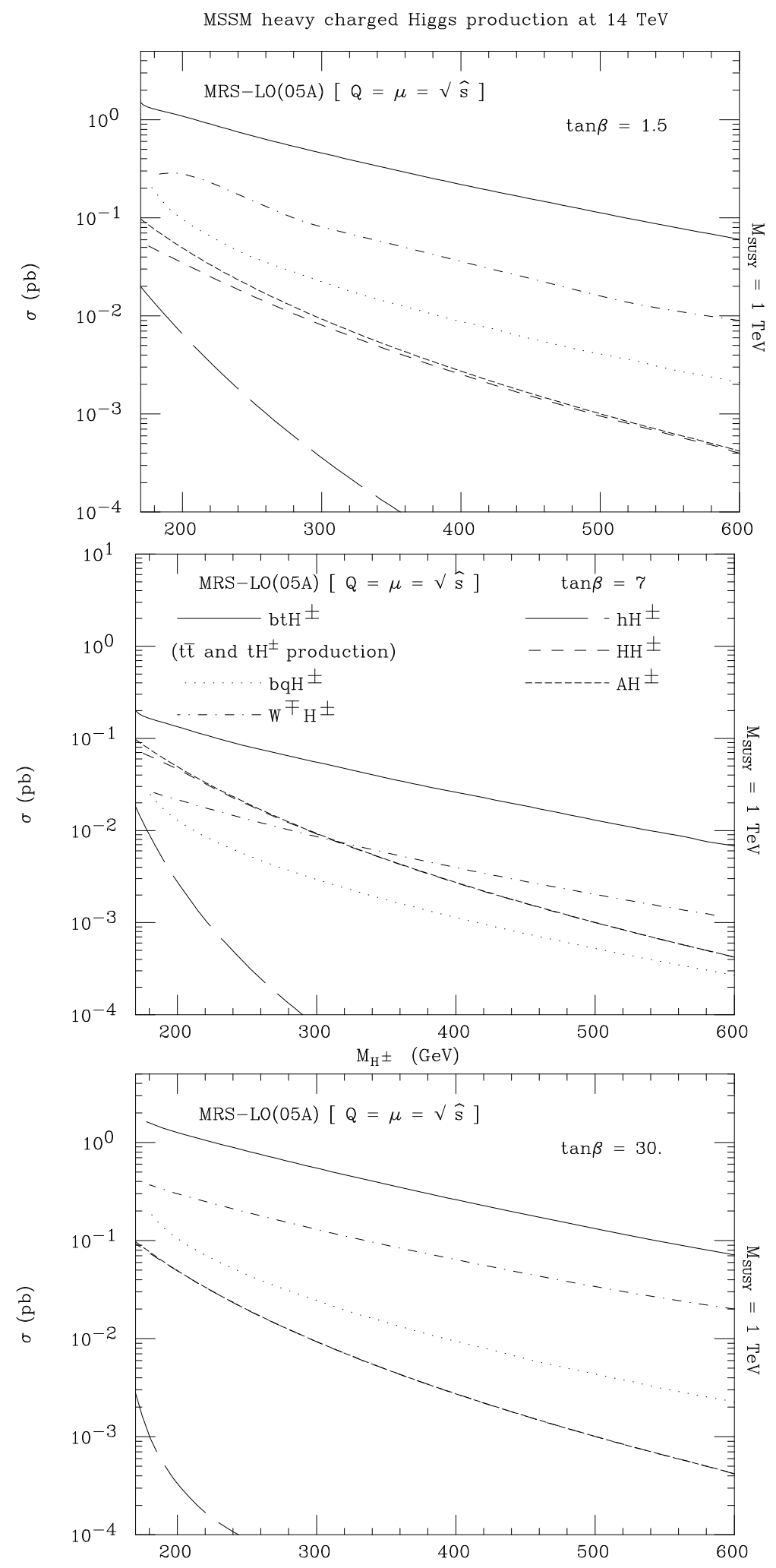

Figure 3: Cross sections in picobarns at the LHC for the production mechanisms of a single charged Higgs boson, for $\tan \beta=1.5$ (top), 7 (middle) and 30 (bottom). (The $q \bar{q}^{\prime} \rightarrow \Phi H^{ \pm}$ rates, with $\Phi=h, A$, visually coincide for $\tan \beta=30$.) 


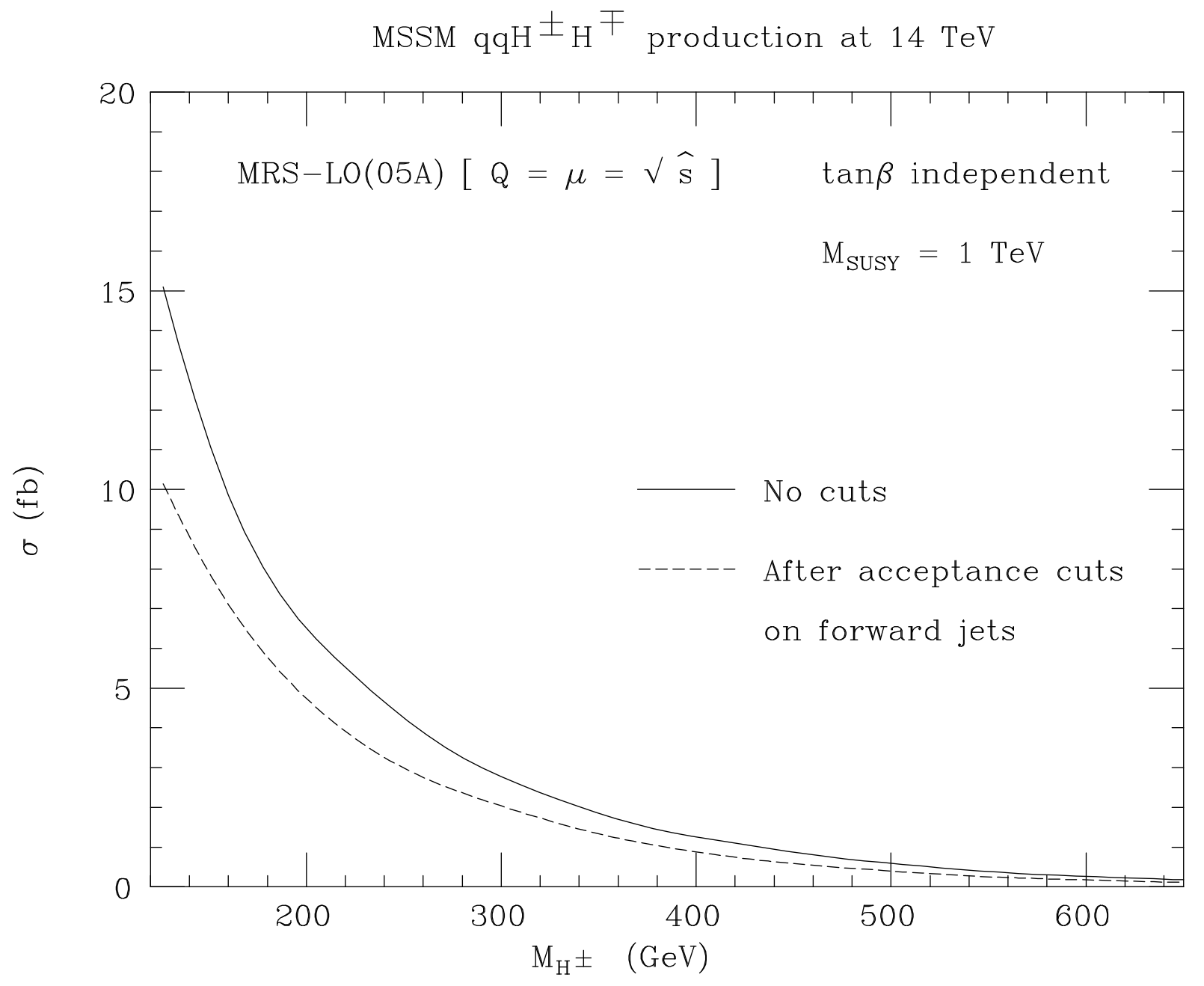

Figure 4: Cross sections in femtobarns at the LHC for process (四), without and with the following acceptance cuts on the forward jets: $p_{T}^{j}>20 \mathrm{GeV}$ and $\left|\eta^{j}\right|<5$. Here, there is no visible $\tan \beta$ dependence. 


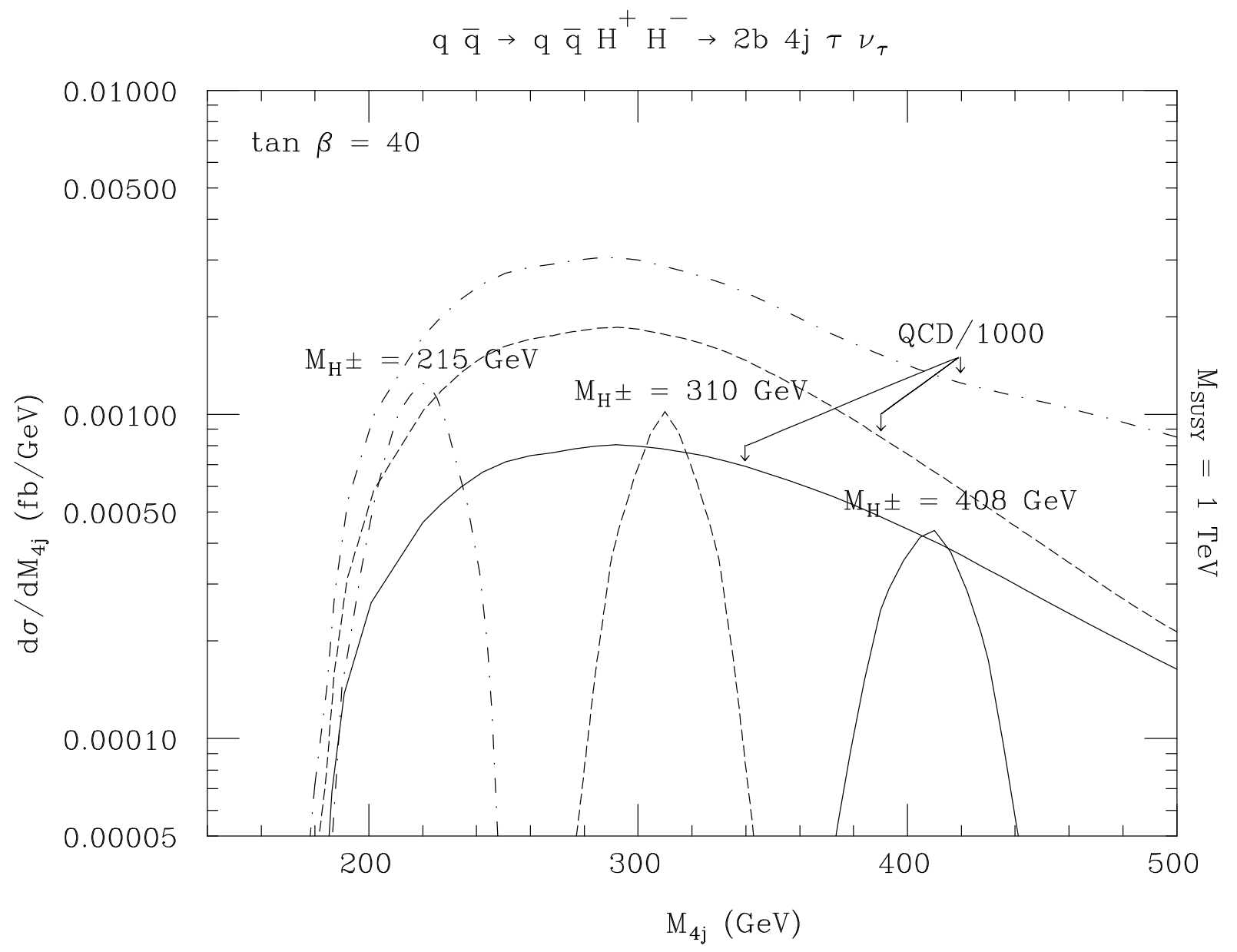

Figure 5: Mass distribution of the four-jet system recoiling against the $j_{1} j_{2}$ and $\tau^{ \pm}$-neutrino pairs, after the selection described in points 2.-8., in case of process (1) and of the QCD background $q \bar{q}, g g \rightarrow t \bar{t} g g$, for three choices of $M_{H^{ \pm}}$in the heavy mass range and $\tan \beta=40$, in the channel ' 6 jets $+\tau^{ \pm}+$missing energy'. 